TITLE:

\title{
Ectopic cervical thymus: A clinicopathological study of consecutive, unselected infant autopsies
}

\section{$\operatorname{AUTHOR}(S)$ :}

Kotani, Hirokazu; Ishida, Tokiko; Miyao, Masashi; Manabe, Sho; Kawai, Chihiro; Abiru, Hitoshi; Omae, Tadaki; Osamura, Toshio; Tamaki, Keiji

\section{CITATION:}

Kotani, Hirokazu ...[et al]. Ectopic cervical thymus: A clinicopathological study of consecutive, unselected infant autopsies. International Journal of Pediatric Otorhinolaryngology 2014, 78(11): 1917-1922

\section{ISSUE DATE:}

2014-11

URL:

http://hdl.handle.net/2433/191111

\section{RIGHT:}

(C) 2014 Elsevier Ireland Ltd.; This is not the published version. Please cite only the published version.; この論文は出版社版でありません。引 用の際には出版社版をご確認ご利用ください。 
Ectopic cervical thymus: A clinicopathological study of consecutive, unselected infant autopsies

Hirokazu Kotani $^{1}$, MD, PhD, Tokiko Ishida ${ }^{1}, \mathrm{MD}$, Masashi Miyao ${ }^{1}, \mathrm{MD}$, PhD, Sho Manabe ${ }^{1}$, MS, Chihiro Kawai ${ }^{1}$, BS, Hitoshi Abiru ${ }^{1}, \mathrm{BS}$, Tadaki Omae ${ }^{2}, \mathrm{MD}$, Toshio Osamura ${ }^{2}, \mathrm{MD}$, PhD, Keiji Tamaki ${ }^{1}, \mathrm{MD}, \mathrm{PhD}$

Affiliations: ${ }^{1}$ Department of Forensic Medicine, Kyoto University Graduate School of Medicine, Yoshida-Konoe-cho, Sakyo-ku, Kyoto 606-8501, Japan; ${ }^{2}$ Department of Pediatrics, Japanese Red Cross Kyoto Daini Hospital, Haruobi-cho 355-5, Kamigyo-ku, Kyoto 602-8026, Japan

Address correspondence to: Hirokazu Kotani. Department of Forensic Medicine, Kyoto University Graduate School of Medicine, Yoshida-Konoe-cho, Sakyo-ku, Kyoto 606-8501, Japan. Telephone: +81 75753 4474; Fax: +81 75761 9591; E-mail:

kotani@fp.med.kyoto-u.ac.jp 


\section{Abstract}

Objectives: An ectopic cervical thymus (ECT) is regarded as a rare congenital anomaly; therefore, the optimal diagnostic and therapeutic strategy remains a debatable matter. We designed a study to elucidate the clinicopathological characteristics of ECTs in consecutive, unselected infant autopsies, to help guide case management.

Methods: We searched for ECTs in all of the 21 consecutive, unselected infant autopsy cases performed at our institution over a period of 3 years, and all ECT consensus diagnoses were confirmed by histological examination. The following clinical characteristics were evaluated in cases with ECTs: age, gender, birth week and weight, clinical symptoms due to the ECT(s), position on discovery of death, cause of death, ECT contribution to the cause of death, and concomitant congenital disorders. The anatomical features evaluated included the location, number, size, color, shape, and margins of the ECTs, and the presence of a mediastinal thymus. Histological findings of the ECT(s) and the mediastinal thymus were compared within each individual. Fusion of the parathyroid and the ECT was also investigated histologically. Spearman's rank correlation coefficient $(\rho)$ and the corresponding $P$ value were calculated to determine if there was an association between ECT diameter and age.

Results: We detected 10 ECT lesions in seven cases (33\%) among the 21 infant autopsy cases. The ECT cases involved five boys and two girls, with ages ranging from 1 day to 4 months. There were no reports of a positive family history of sudden death or antemortem clinical symptoms due to ECT in any of the cases. The ECTs were considered incidental regarding the cause of death, with the exception of one case that was extremely rare. Most ECTs were 
localized to the inferior thyroid, ranging from 0.4 to $1.9 \mathrm{~cm}$ in size. Size demonstrated a significant negative correlation with age $(\rho=0.75$ and $P=0.034)$.

Conclusions: This study revealed that ECT is an essentially benign anomaly that occurs frequently during the development of the thymus, and may disappear over the first few years of life. These results suggest a conservative approach to the management of ECTs would be appropriate.

\section{Keywords:}

Ectopic cervical thymus, Natural incidence, Clinical course, Infant, Sudden unexpected death in infancy, Congenital anomaly 


\section{Introduction}

An ectopic cervical thymus (ECT), which can be located anywhere along the developmental pathway of thymic descent, represents a rare cause of pediatric neck masses [1-3]. The natural incidence and clinical course of ECTs are still unknown because nearly all of the previously reported cases have undergone surgical removal, and a prospective randomized study is not feasible because of the rarity of ECTs [4]. This lack of knowledge has led to controversy regarding the optimal diagnostic and therapeutic strategy for this lesion $[4,5]$. Some reports have recommended surgical excision based on the safety of this procedure, the necessity for a pathological diagnosis, and the potential for malignant transformation and severe clinical symptoms such as dyspnea and dysphagia without surgery $[2,6,7]$. Meanwhile, others have recommended conservative management, such as a "watch and wait” policy, for ECTs diagnosed before surgery because of concerns regarding the high morbidity associated with surgical excision; these proponents consider ECT to be an anomaly with a basically benign course and without convincing evidence to support an increased risk of malignant transformation $[4,8]$.

Advanced high-resolution ultrasonography (US) and magnetic resonance imaging (MRI) have recently been described as alternatives to histological evaluation for the diagnosis of an ECT [9-11]. Song et al. stated that the echo characteristics of ECT, such as multiple echogenic inner linear structures and foci surrounded by hypoechoic rims, are key to its diagnosis and that MRI facilitates diagnosis because the appearance of an ECT is identical to that of a mediastinal thymus on MRI [9]. In addition to these imaging methods, fine needle 
aspiration has been recommended as a diagnostic method in recent years because it is a less invasive procedure than surgical excision; the presence of fibrous septa, Hassall’s corpuscles, and other characteristics of normal thymic architecture on cytology can provide clues to an accurate diagnosis of ECT $[4,8,11,12]$.

If we could improve current diagnostic strategies by increasing available information regarding the natural incidence and clinical course of ECTs, it would help pediatricians to determine the appropriate strategy when they encounter a particular case that may require surgery. A prospective randomized study with healthy infants using radiological images such as US may seem to be a logical approach. However, the images do not provide a definitive diagnosis of ECT without pathological confirmation. Therefore, we decided to investigate the clinicopathological characteristics of ECTs by performing a survey of consecutive, unselected infant autopsies associated with histological examinations. To the best of our knowledge, this study is the first to clarify the features of this lesion in consecutive autopsies at a single institution.

\section{Materials and Methods}

\subsection{Cases}

We searched for ECTs in all 21 of the consecutive, unselected infant cases autopsied at our institution for 3 years from the time we encountered the first ECT case, which we 
previously described [3]. The 21 cases were categorized into sudden unexpected death in infancy (SUDI) and ranged in age from 1 day to 10 months; 13 of the cases were boys. No cases required exclusion because of unavailability, e.g., because of a long postmortem interval or severe neck trauma. The Department of Forensic Medicine at Kyoto University Graduate School of Medicine, Japan, is one of two forensic autopsy institutions in the Kyoto prefecture (population of 2.8 million) and is assigned two-thirds of the out-of-hospital SUDI cases. The following clinical characteristics were evaluated in cases with ECTs: age, gender, birth week and weight, clinical symptoms due to the ECT(s), position on discovery of death, cause of death, ECT contribution to the cause of death, and concomitant congenital disorders. This study was conducted within the framework established by the Ethics Committee of Kyoto University.

\subsection{Anatomical and histological features}

In all cases, the cervical organs along with the tongue and thoracic organs were resected after removal of the heart. Two experienced forensic pathologists (H.K and K.T) sought ECTs throughout the cervical and thoracic organs, and all ECT consensus diagnoses were confirmed by histological examination. The anatomical features evaluated included the location, number, size, color, shape, and margins of the ECTs and the presence of a mediastinal thymus. Size was defined as the greatest diameter. Histological findings of the ECT(s) and mediastinal thymus were compared within each individual on H\&E stained 
specimens and by immunohistochemical analysis of the lymphocytes. Fusion of the parathyroid and the ECT was also investigated histologically.

\subsection{Statistical analyses}

Spearman's rank correlation coefficient $(\rho)$ and the corresponding $P$ value were analyzed to determine if there was an association between diameter and age. $P$ value less than 0.05 was considered statistically significant. All statistical analyses were performed using R software for Windows, version 2.14.1.

\section{Results}

\subsection{Clinical characteristics of cases with ECT}

We encountered seven cases with solid ECTs in 21 consecutive, unselected infant autopsies during the study period, an incidence of 33\% within this population. The clinical characteristics of the seven cases are summarized in Table 1; the cases ranged in age from 1 day to 4 months, and five cases (71\%) in this series were boys. All were born as mature full term infants except for one male infant with congenital heart disease born at a gestational age of 36 weeks. There were no reports of a positive family history of sudden death or antemortem clinical symptoms due to ECT in any of the cases. Five were found dead in a 
supine position, and two were found in a prone position. The cause of death was sudden infant death syndrome in three cases, pneumonia due to respiratory syncytial virus infection in two, and accidental asphyxia in one. The ECTs were considered to have been incidental to their deaths with the exception of a 4-month-old boy (case 6).

\subsection{Anatomical features of the ECTs}

Anatomical features of the ECTs are summarized in Table 2. The ECTs were found on both sides without laterality but were preferentially located at the inferior region of the thyroid gland except for one visually undetectable ECT. The detectable ECTs appeared as well-circumscribed soft and solid nodules with a spherical shape and a reddish gray-white color (Figs. 1a, b; Supplementary Fig. 1). The undetectable lesion was covered by the right lobe of the thyroid, and the thyroid isthmus was absent (Fig. 1b, case 7); the ECT was detected on histological examination (Figs. 1b, 2a). Three cases had two lesions each; the others had a single lesion. The mean lesion size was $1.0 \mathrm{~cm}$ (range, 0.4 to $1.9 \mathrm{~cm}$ ). The size of the lesion demonstrated a significant negative correlation with age for all ECTs except the two lesions found in case 6 (Fig. 1c). Spearman's rank correlation coefficient $(\rho)$ thas 5 and the corresponding $P$ value for the correlation was 0.034 . Normal mediastinal thymuses were present in all seven cases.

\subsection{Histological features of the ECTs}


Histological examination demonstrated that the ECTs located outside of the thyroid gland were surrounded by a thin fibrous capsule, while the ECT covered by the thyroid gland was located immediately adjacent to the gland but was clearly separated from it by thin fibrous tissue (Fig. 2a, b). Five ECTs from three cases were fused with the parathyroid, and these lesions looked like single rounded nodules because both the ECT and the parathyroid were enveloped in a continuous fibrous capsule or tissue (Fig. 2a). Four of the five parathyroids fused with ECTs were located at the periphery of the lesion (as shown by the arrows in Fig. 2) and another was located at the center of the lesion (data not shown). All parathyroids occupied one-third to one-fourth of the associated ECT (Fig. 2a, b). Histological features of ECTs demonstrated on H\&E stain and immunohistochemistry were consistent with ordinary thymic structure, and thymocytes and lymphocytes were typically distributed (Fig. 2b, c).

\section{Discussion}

ECT results from a failure of thymic descent during weeks 6 and 8 of pregnancy [13]. The primordia of the thymus and inferior parathyroids are mainly derived from the ventral and dorsal wings of the third pharyngeal pouch, respectively; a lesser portion of the thymus is derived from the fourth pharyngeal pouch. The thymic primordia then elongate, along with the inferior parathyroids, in a caudal and medial direction, forming a structure called the thymopharyngeal duct. During the descent into the anterosuperior mediastinum, a tail portion 
of the thymus falls into pieces and eventually vanishes under normal conditions, but during this process, the primordia may arrest in the duct to form an ECT $[9,13,14]$. Because the inferior parathyroids descend with the thymus, it is not surprising that they are frequently found within ECTs [13, 15-17]; indeed, half of the 10 ECTs found in the present study were fused with the parathyroids.

To estimate the natural incidence of ECTs, we surveyed consecutive, unselected infant autopsies and definitively identified ECT lesions based on histological examination. The study yielded an incidence of approximately 30\%, which is assumed to be close to the natural incidence because of the nonselective method employed. Accordingly, we can speculate that this anomaly occurs more frequently than would be expected based on previous reports (the incidence from previous reports ranges between $1 \%$ and 10\%) [6, 17-19]. This discrepancy between previous reports and the present study may be attributed to the different populations analyzed $[3,19]$. Most previous reports represent case presentations or reviews consisting of several cases $[14,20]$, with almost all of the cases showing clinical symptoms, such as a neck mass, due to the anomaly [3]. In contrast, most of the ECTs in our study represented incidental findings identified in infants who died from unrelated causes. These results indicate that ECTs occur frequently during thymus development, and usually remain undetected, as only some ECTs increase in size and induce clinical symptoms.

In fact, some previous studies have also suggested the potential prevalence of this lesion [9, 17]. However, there is no report confirming the commonness in the unselected cases with supporting histological examinations as presented in the current study. For 
example, Song et al. investigated the imaging of ECT, and stated that the frequency of subclinical ECTs may be underestimated, because they found several incidental asymptomatic cases in addition to the cases analyzed during their study [9]. They did not confirm, however, their diagnosis of ECTs with histological examination. In another report, ECTs were found in 39 of 55 SUDI autopsies (71\%) conducted under a stereoscopic microscope, although this reported incidence may be higher than the incidence in our study due to their selection bias [17]. Nonetheless, their data supports our results that this anomaly may arise more frequently than reported, and probably occurs in more than $30 \%$ of infants.

Assuming that ECT weight changes with age like that of the mediastinal thymus, the ECT would steadily increase in size until puberty and then gradually decrease in size with involution [13]. However, in a correlation analysis among the asymptomatic cases in this study, a significant negative correlation was observed between ECT size and age even within infancy (Fig. 1c, $\rho=-0.75 ; P<0.05$ ). The chart in Fig. 1c also suggests the possibility that the ECT is not often detected in children after the first year of life, which is in accord with results we have recently reported in a literature review of 95 cases with ECT(s) [3]. Most of the cases analyzed in the review (82\%) were diagnosed in patients less than one year of age, and the majority of the cases included the manifestation of symptoms [3]. These results indicate that most of the ECTs, even among the symptomatic cases, are detected within infancy, but seldom after the first year of life. In light of this trend towards a reduction in size and rarity in children aged one or older, when pediatricians find an asymptomatic ECT or a small neck mass without symptoms, they could assume that the ECT will disappear at some 
point in the near future. The mechanism of size reduction is unclear but may represent a process other than involution such as circulatory insufficiency [21], because smaller ECTs in older infants did not show the histological features typical of thymic involution, i.e., a decrease in the number of thymocytes and abundant intervening adipose tissue (e.g., case 7 in Fig. 2) [13].

Thus, the ECT most often has an indolent course, and can be managed conservatively. However, some previous case reports have described the enlargement of ECTs during various periods, along with the induction of symptoms [18, 22-25]. Our previous review of 95 cases revealed that relatively severe symptoms, such as respiratory symptoms and/or dysphagia, are seen in approximately $10 \%$ of the reported cases [3]. Indeed, the two ECTs presented in case 6 were large and highly displaced from the correlation chart (Fig. 1c). Consequently, they may have contributed to the accidental asphyxia in the prone position because their increased size and specific location allowed easy compression of the cervical trachea, as was described in detail in a previous report (Suppl. Fig.) [3]. However, such cases leading to fatality are extremely rare, and only two cases have been reported to date [3, 26]. Pediatricians who discover symptomatic ECT(s) would understandably be cautious, and should monitor the progression of the ECT closely. Nevertheless, the current results suggest that the worst outcomes are unusual, and do not occur in most cases.

The mechanism of enlargement is not yet clear, but prior reports have described potential hyperplasia of ECTs following infection or vaccination [6, 25, 27-30]. Indeed, the infant in case 6 received a second combination vaccine against diphtheria, pertussis, and 
tetanus two days before his death. However, of the other cases in this study, the two cases that died from pneumonia (cases 4 and 7), and the two cases that were vaccinated within one month prior to death (cases 4 and 5) did not present with large ECTs. Nonetheless, the present study was not designed to reveal whether an ECT would enlarge because of an inflammatory reaction triggered by infection or vaccination, and thus further studies are required to clarify this issue.

We recently reviewed the clinicopathological characteristics of 95 ECT cases [3], and analysis of the seven cases identified in the present study revealed similar clinical features (Table 1). In brief, ECTs are frequently seen in boys and are typically diagnosed at < 6 months of age (70\%); no apparent propensity has been demonstrated among the causes of death; symptoms such as respiratory symptoms and/or dysphagia are seen in $12 \%$ of cases, but ECT contribution to severely ill or death is extremely rare; and approximately one-third of patients with ECTs have a concomitant congenital anomaly/disorder, but no specific anomalies are strongly associated with ECTs.

As for the pathological features of ECTs, we have presented seven macroscopic photographs in our present and previous reports (supplementary figure) demonstrating the following common gross findings: a soft nodule with a reddish gray-white color, a clear circumference, and a spherical shape. These characteristic gross findings may aid surgeons and pathologists in identifying ECTs during operations and autopsies, respectively. In this study, all ECTs except for one were found in the inferior region of the thyroid gland (Table 2), although the localization of lesions to this site was relatively rare (13\%) among the 95 cases 
previously described [3]. The predisposition for the inferior thyroid region identified in the present study may indicate that the inferior parathyroids frequently pull part of the thymus into an adjacent position during thymic descent to form an associated ECT, but that these ECTs seldom induce clinical symptoms because of their disappearance in infancy. We confirmed all ECTs on histology and found that the structure and cell distribution of the ECTs did not differ from that of the mediastinal thymus, which correlates with the previous literature [5, 6, 15-17, 31].

These seven ECT cases represent the first case series of consecutive infant autopsies performed at a single institution. However, the small number of cases limited to autopsies performed for SUDI is a limitation of our study. A prospective randomized study in a larger population of healthy infants utilizing diagnostic imaging techniques, especially high-resolution US with fine needle aspiration, would be the preferred approach for clarifying the natural features of ECTs, but is not feasible in practice.

\section{Conclusions}

This study is the first to clarify the features of ECT in consecutive, unselected infant autopsies at a single institution, and revealed that this lesion is an essentially benign anomaly that occurs frequently during the development of the thymus, and may disappear over the first few years of life. These results suggest a conservative approach to the management of ECTs would be appropriate. 


\section{Conflict of interest}

The authors declare that they have no conflicts of interest.

\section{Sources of funding}

The authors have no financial relationships with any organization that might have an interest in the submitted work. No external funding was secured for this study.

\section{Author's contributions}

Hirokazu Kotani: Dr. Kotani conceptualized and designed the study, performed the autopsies, collected and interpreted the data, and wrote the manuscript.

Tokiko Ishida, Masashi Miyao, Chihiro Kawai, Hitoshi Abiru: Dr. Ishida and Miyao, BSs Kawai and Abiru participated in the autopsies and preparation of the histopathological specimens, and approved the final manuscript.

Sho Manabe: MS. Manabe performed the statistical analyses, and approved the final manuscript.

Tadaki Omae, Toshio Osamura: Drs. Omae and Osamura attended the case in the emergency room, and approved the final manuscript.

Keiji Tamaki: Dr. Tamaki performed the autopsies, performed a critical revision of the manuscript for important intellectual content, and gave final approval for submission of this version for publication. 


\section{References}

[1] Tovi F, Mares AJ, The aberrant cervical thymus: Embryology, pathology, and clinical implications, The American Journal of Surgery,136 (1978) 631-637.

[2] Khariwala SS, Nicollas R, Triglia JM, Garabedian EN, Marianowski R, Van Den Abbeele T, April M, Ward R, Koltai PJ, Cervical presentations of thymic anomalies in children, International Journal of Pediatric Otorhinolaryngology,68 (2004) 909-914.

[3] Ishida T, Kotani H, Miyao M, Abiru H, Kawai C, Osamura T, Tamaki K, Ectopic cervical thymus associated with infant death: 2 case reports and literature review, International Journal of Pediatric Otorhinolaryngology,77 (2013) 1609-1612.

[4] Schloegel LJ, Gottschall JA, Ectopic cervical thymus: is empiric surgical excision necessary?, Int J Pediatr Otorhinolaryngol,73 (2009) 475-479.

[5] Zhaoqiang Z, Qingbin Z, Lei C, Dan C, Yu C, Wangxiang Y, Xueqiang D, Infant ectopic cervical thymus one case report: Diagnostic and management difficulties, Journal of Cranio-Maxillofacial Surgery,40 (2012) 701-705.

[6] Wang J, Fu H, Yang H, Wang L, He Y, Clinical management of cervical ectopic thymus in children, Journal of Pediatric Surgery,46 (2011) e33-e36.

[7] Mizia-Malarz A, Sobol G, Maldyk J, Stolpa W, Szyszka A, Wos H, Cervical ectopic thymus in a 9-month-old girl: diagnostic difficulties, J Pediatr Hematol Oncol,31 (2009) 599-601. 
[8] Tunkel DE, Erozan YS, Weir EG, Ectopic cervical thymic tissue: diagnosis by fine needle aspiration, Arch Pathol Lab Med,125 (2001) 278-281.

[9] Song I, Yoo SY, Kim JH, Hong E, Yoon HK, Aberrant cervical thymus: imaging and clinical findings in 13 children, Clinical Radiology,66 (2011) 38-42.

[10] Slovis TL, Meza M, Kuhn JP, Aberrant thymus--MR assessment, Pediatr Radiol,22 (1992) 490-492.

[11] Zielke A, Swischuk L, Hernandez JA, Ectopic cervical thymic tissue: can imaging obviate biopsy and surgical removal?, Pediatric Radiology,37 (2007) 1174-1177.

[12] Taweevisit M, Sampatanukul P, Thorner PS, Ectopic thymoma can mimic benign and malignant thyroid lesions on fine needle aspiration cytology: a case report and literature review, Acta Cytol,57 (2013) 213-220.

[13] Mills SE, Histology for Pathologist. 4th ed. City: Lippincott Williams \& Wilkins, 2012; 541-542.

[14] Spigland N, Bensoussan AL, Blanchard H, Russo P, Aberrant cervical thymus in children: three case reports and review of the literature, J Pediatr Surg,25 (1990) 1196-1199.

[15] Daneshbod Y, Banani A, Kumar PV, Aberrant thymus and parathyroid gland presenting as a recurrent lateral neck mass: a case report, Ear Nose Throat J,85 (2006) 452-453.

[16] Schramm JC, Perry DA, Sewell RK, Retropharyngeal thymus and parathyroid gland: A case report, International Journal of Pediatric Otorhinolaryngology,78 (2014) 163-165.

[17] Ellis HA, Knight B, Parathyroids and cervical thymus in sudden unexpected death in infancy, Pediatrics,44 (1969) 225-233. 
[18] Clark JJ, Johnson SM, Solid cervical ectopic thymus in an infant, J Pediatr Surg,44 (2009) e19-21.

[19] Bale PM, Sotelo-Avila C, Maldescent of the thymus: 34 necropsy and 10 surgical cases, including 7 thymuses medial to the mandible, Pediatr Pathol,13 (1993) 181-190.

[20] Nowak PA, Zarbo RJ, Jacobs JR, Aberrant solid cervical thymus, Ear Nose Throat J,67 (1988) 670, 673, 676-677.

[21] Kumar V, Abbas AK, Fausto N, Aster JC, Robbins and Cotran Pathologic Basis of Disease 8th ed. City: Saunders Elsevier, 2010.

[22] McLeod DM, Karandy EJ, Aberrant cervical thymus. A rare cause of acute respiratory distress, Arch Otolaryngol,107 (1981) 179-180.

[23] Michelson H, Sender B, Cervical thymus; report of a case, AMA Arch Surg,72 (1956) 275-276.

[24] Kacker A, April M, Markentel CB, Breuer F, Ectopic thymus presenting as a solid submandibular neck mass in an infant: case report and review of literature, International Journal of Pediatric Otorhinolaryngology,49 (1999) 241-245.

[25] Felgentreff K, Schupp W, Otten J, Rückauer K, Uhl M, Jüttner E, Superti-Furga A, Pohl M, Inspiratory stridor and dysphagia in two newborn infants caused by ectopic thymus tissue, European Journal of Pediatrics,168 (2009) 1141-1145.

[26] Weissman I, Smith WC, Accessory thymus (paratracheal) with tracheal obstruction, Ill Med J,112 (1957) 23-26.

[27] Loney DA, Bauman NM, Ectopic cervical thymic masses in infants: A case report and 
review of the literature, International Journal of Pediatric Otorhinolaryngology,43 (1998) 77-84.

[28] Wagner CW, Vinocur CD, Weintraub WH, Golladay ES, Respiratory complications in cervical thymic cysts, Journal of Pediatric Surgery,23 (1988) 657-660.

[29] Saggese D, Ceroni Compadretti G, Cartaroni C, Cervical ectopic thymus: a case report and review of the literature, International Journal of Pediatric Otorhinolaryngology,66 (2002) 77-80.

[30] Scott KJ, Schroeder AA, Greinwald JH, Jr, Ectopic Cervical Thymus: An Uncommon Diagnosis in the Evaluation of Pediatric Neck Masses, Arch Otolaryngol Head Neck Surg,128 (2002) 714-717.

[31] Shah SS, Lai SY, Ruchelli E, Kazahaya K, Mahboubi S, Retropharyngeal Aberrant Thymus, Pediatrics,108 (2001) e94. 


\section{Figure legends}

Fig. 1a, b: Representative gross appearance of the resected specimens showing two nodules (a, case 2) and one nodule (b, case 7) located beneath the thyroid gland. \#, thyroid gland; *, ectopic cervical thymus (ECT). c: Plot demonstrating the significant negative correlation observed between ECT size and infant age (Spearman's rank correlation coefficient $(\rho)=$ -0.75 and $P=0.034)$. Numbers inside circles indicate the case numbers listed in Tables 1 and 2.

Fig. 2 Representative microscopic ECT appearance (case 7). a: Loupe image showing two lesions, one on each side of the cervical bronchus; the ECT on the left is located outside of the thyroid gland, and the ECT on the right is covered by the gland. b: High-magnification image of the right lesion. Arrows, parathyroid gland; asterisks, ECT. c: Immunohistochemical images of ECT and mediastinal thymus specimens showing no difference in the distribution of lymphocytes; CD3 and CD20 staining indicate the distribution of T cells and B cells, respectively. 

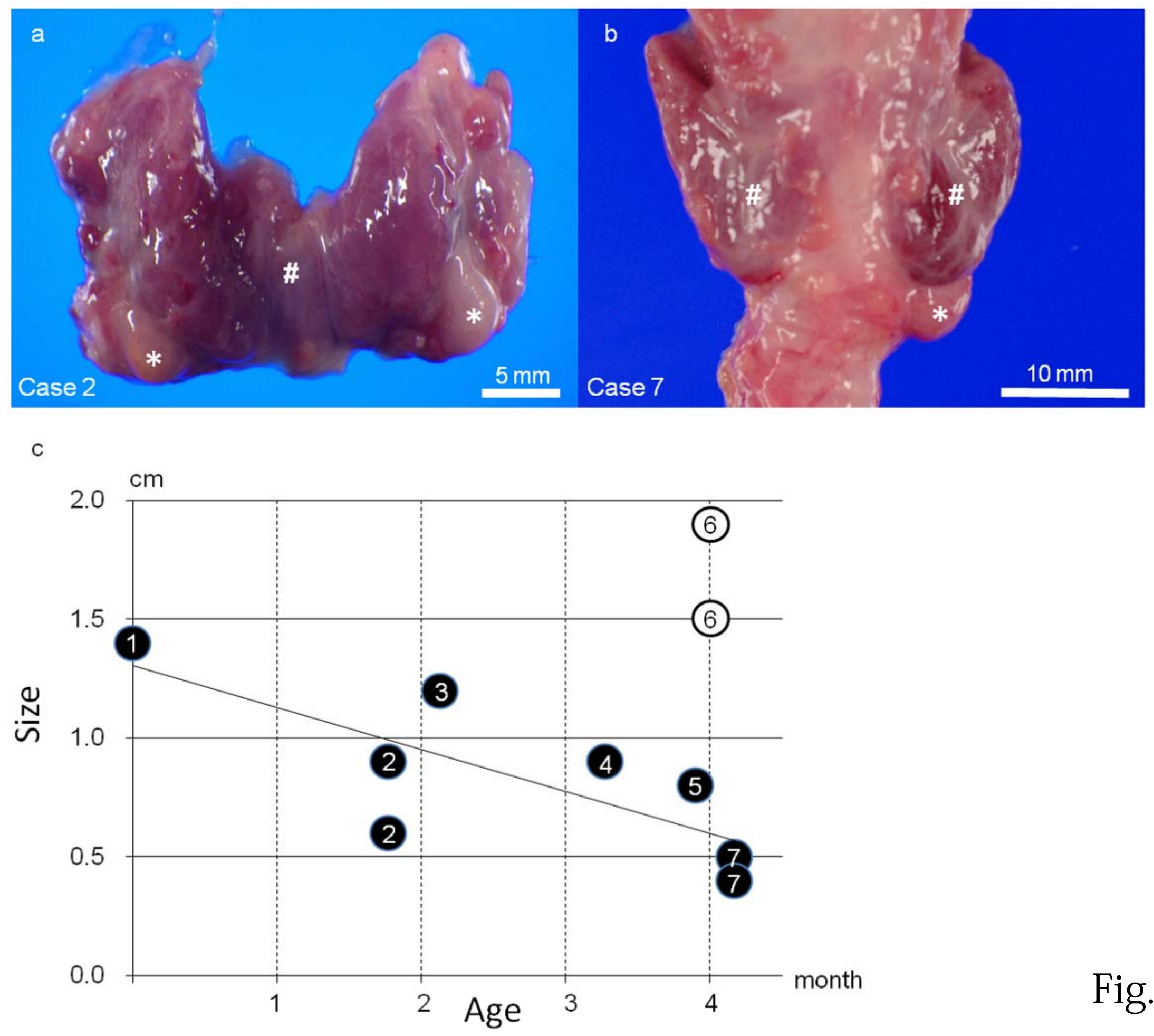

Fig. 1 

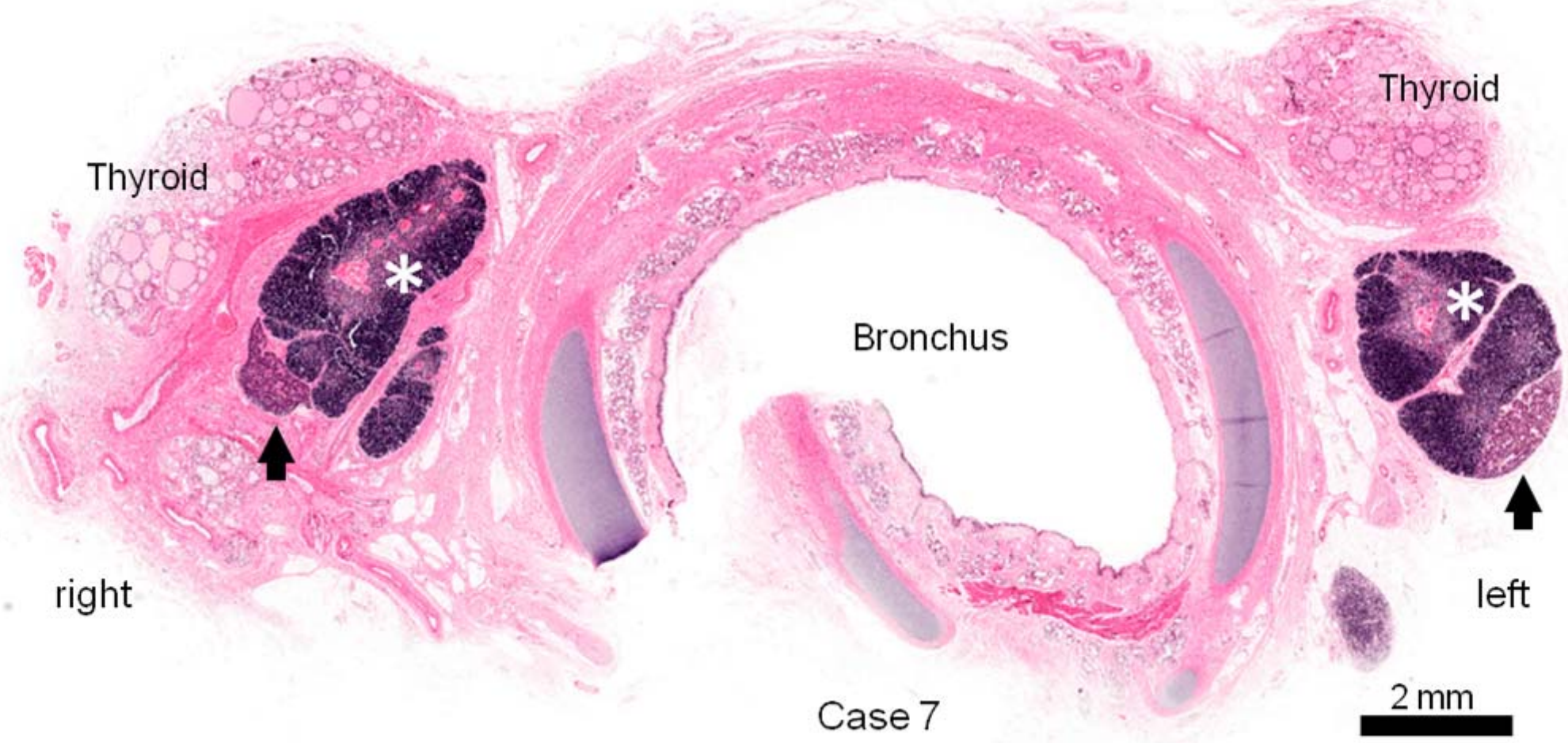

b
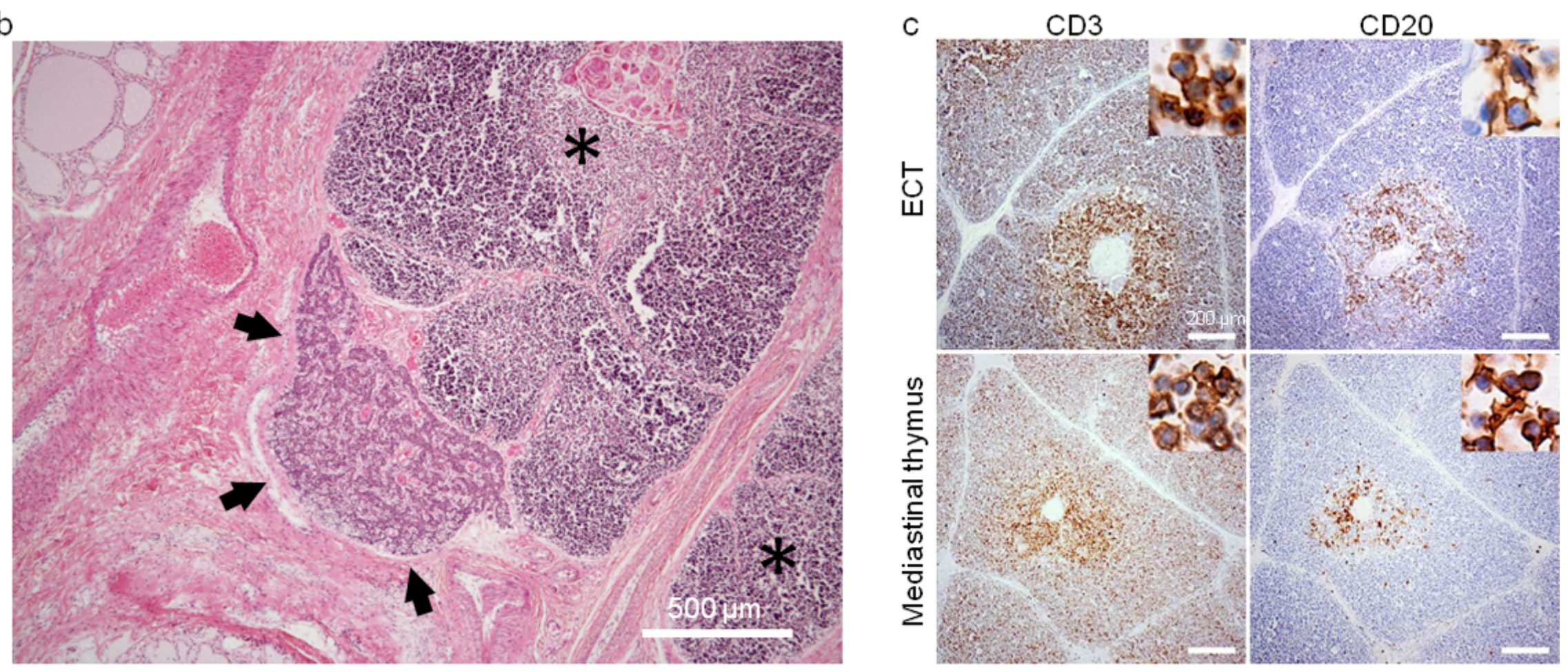

Fig. 2 
Table 1 Clinical characteristics of cases with ectopic cervical thymuses

\begin{tabular}{cccllll}
\hline $\begin{array}{l}\text { Case } \\
\text { No. }\end{array}$ & Age / Gender & $\begin{array}{l}\text { Birth } \\
\text { wk / wt (g) }\end{array}$ & $\begin{array}{l}\text { Position on } \\
\text { discovery }\end{array}$ & $\begin{array}{l}\text { Cause of } \\
\text { death }\end{array}$ & $\begin{array}{l}\text { ECT contribution } \\
\text { to death }\end{array}$ & $\begin{array}{l}\text { Congenital } \\
\text { anomalies }\end{array}$ \\
\hline 1 & 1 day /M & $36 / 2,300$ & Supine & CCA & No & CCA \\
2 & 2 months/M & $40 / 3,250$ & Supine & SIDS & No & Absent \\
3 & 2 months/ F & $40 / 3,210$ & Supine & SIDS & No & Absent \\
4 & 3 months/M & $38 / 3,310$ & Prone & Pneumonia & No & Absent \\
5 & 4 months/M & $37 / 2,410$ & Supine & SIDS & No & Absent \\
6 & 4 months/M & $40 / 3,472$ & Prone & Asphyxia & Yes & Absent \\
7 & 4 months/ F & $39 / 2,888$ & Supine & Pneumonia & No & ATI \\
\hline
\end{tabular}

ATI, absent thyroid isthmus; CCA, congenital cardiac anomaly; ECT, ectopic cervical thymus;

SIDS, sudden infant death syndrome. 
Table 2 Anatomical features of ectopic cervical thymuses

\begin{tabular}{|c|c|c|c|c|c|c|}
\hline \multirow{2}{*}{$\begin{array}{l}\text { Case } \\
\text { No. }\end{array}$} & \multicolumn{2}{|c|}{ Location } & \multirow[t]{2}{*}{ Number } & \multirow{2}{*}{$\begin{array}{l}\text { Size } \\
(\mathrm{cm})\end{array}$} & \multirow{2}{*}{$\begin{array}{l}\text { Mediastinal } \\
\text { thymus }\end{array}$} & \multirow{2}{*}{$\begin{array}{l}\text { Parathyroid } \\
\text { gland }\end{array}$} \\
\hline & $\mathrm{L} / \mathrm{R}$ & Level & & & & \\
\hline 1 & $\mathrm{~L}$ & $\operatorname{Inf}^{\mathrm{a}}$ & 1 & 1.4 & Normal & Not detected \\
\hline 2 & $\mathrm{~L} / \mathrm{R}$ & Inf & 2 & $0.9,0.6$ & Normal & Detected in both \\
\hline 3 & $\mathrm{R}$ & Inf & 1 & 1.2 & Normal & Not detected \\
\hline 4 & $\mathrm{R}$ & Inf & 1 & 0.9 & Normal & Detected \\
\hline 5 & $\mathrm{~L}$ & Inf & 1 & 0.8 & Normal & Not detected \\
\hline 6 & $\mathrm{~L} / \mathrm{R}$ & Inf & 2 & $1.5,1.9$ & Normal & Not detected \\
\hline 7 & $\mathrm{~L} / \mathrm{R}$ & Inf/Thy ${ }^{\mathrm{b}}$ & 2 & $0.5,0.4$ & Normal & Detected in both \\
\hline
\end{tabular}

a "Inf" indicates localization of the ECT to the inferior region of the thyroid.

b "Thy" indicates that the ECT is surrounded by the thyroid. 


\section{Gross appearances of the ECTs}
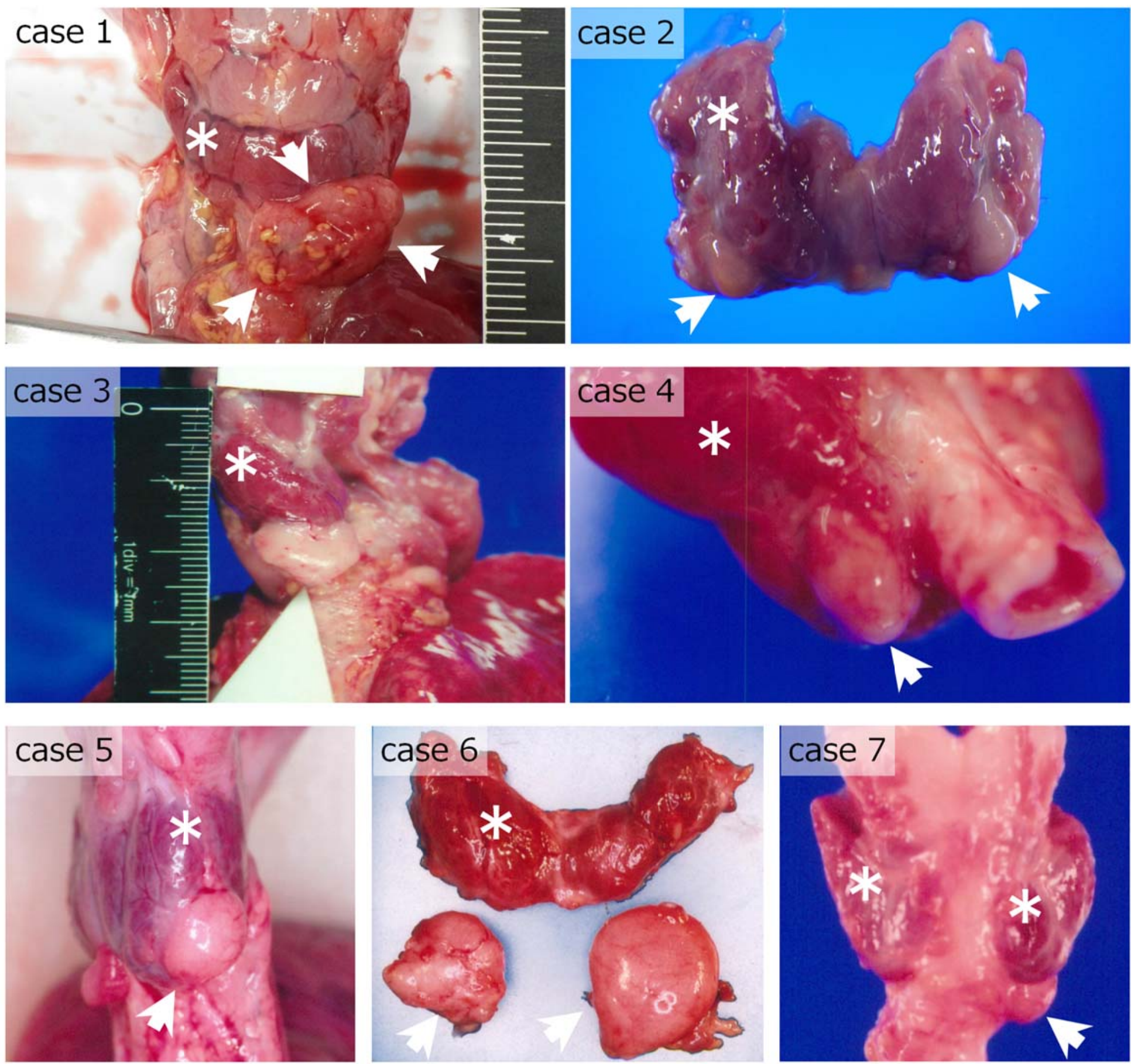

White arrows indicate ECTs; asterisks indicate thyroids. 